\title{
Combined GM-CSF treatment and M-CSF inhibition of tumor-associated macrophages induces dendritic cell-like signaling in vitro
}

\author{
YUSUKE KITOH $^{1}$, MASANAO SAIO ${ }^{4}$, NAOE GOTOH ${ }^{1}$, NAOKI UMEMURA ${ }^{2}$, \\ KENICHI NONAKA ${ }^{3}$, JUNCHENG BAI ${ }^{1}$, LAURA VIZKELETI ${ }^{5}$, DANIEL TOROCSIK ${ }^{5}$, \\ MARGIT BALAZS $^{5}$, ROZA ADANY ${ }^{5}$ and TSUYOSHI TAKAMI ${ }^{1}$
}

\begin{abstract}
Departments of ${ }^{1}$ Immunopathology, ${ }^{2}$ Oral and Maxillofacial Sciences, and ${ }^{3}$ Sugical Oncology, Gifu University Graduate School of Medicine, Gifu; ${ }^{4}$ Pathology Division, University Hospital, University of the Ryukyus, 207 Aza-Uehara, Nishihara-cho, Nakagami-gun, Japan; ${ }^{5}$ Department of Preventive Medicine, University of Debrecen, Hungary
\end{abstract}

Received December 3, 2010; Accepted January 21, 2011

DOI: $10.3892 /$ ijo.2011.960

\begin{abstract}
Macrophages demonstrate plasticity, and tumorassociated macrophages (TAM) can function as immunosuppressive cells in the tumor microenvironment. Therefore, in this study, we aimed to reprogram TAM in vitro with cytokine signal alteration. Granulocyte macrophage colony stimulating factor (GM-CSF) treatment alone did not lead to changes in the expression of M1 (including IL-1 $\beta, T N F \alpha$ and CXCL-10) or M2 (including CD36, CD206 and CCL17) molecules by TAM in vitro, although they adopted a round morphology and were less adhesive to the culture dish. When macrophage colony stimulating factor (M-CSF) signals were suppressed by siRNA against the M-CSF receptor (M-CSFR) in conjunction with GM-CSF treatment, the signal transduction pathway of TAM was altered, and the expression of STAT1, STAT5 and STAT6, which are usually expressed by dendritic cells, was increased. However, the same treatment did not alter the TAM expression pattern of M1/M2 marker molecules. With respect to the NF-kB pathway, GM-CSF and M-CSFR siRNA combination treatment significantly induced the expression of p65, which is usually not expressed by TAM, while p50 and p105 expression by TAM was not affected by the treatment. These findings indicate that our model could not redirect TAM to a monocyte-derived dendritic cell-like phenotype based on the analysis of M1/M2 marker expression, but it was able to modify cell signaling pathways toward a dendritic cell-like pattern. Therefore, the present data suggest that TAM demonstrate plasticity toward dendritic cell-like
\end{abstract}

Correspondence to: Dr Yusuke Kitoh, Department of Immunopathology, Gifu University Graduate School of Medicine, Yanagido, Gifu city, Gifu 501-1194, Japan

E-mail: m2111701@edu.gifu-u.ac.jp

Key words: tumor-associated macrophages, granulocyte-macrophage colony stimulating factor, macrophage colony stimulating factor receptor, cell signaling signal transduction patterns, and that the alteration of the tumor microenvironment has the potential to reverse the immunosuppressive properties of TAM.

\section{Introduction}

Tumor-infiltrating myeloid cells are immature cells that are activated and matured under the influence of the tumor microenvironment and composed of two main subtypes, granulocytes and monocytes/macrophages $(1,2)$, which have immunosuppressive properties (3). Among these cells, $\mathrm{CD} 11 \mathrm{~b}^{+} \mathrm{Gr}^{+}{ }^{+}$ granulocyte-lineage cells, also referred to as myeloid-derived suppressor cells $(4,5)$, are present in bone marrow, spleen, and tumor tissue of the tumor-bearing host and suppress T-cell functions (6-9). In contrast, tumor-infiltrating monocyte/macrophage-lineage cells, referred to as tumor-associated macrophages (TAM), show immunosuppressive properties (10).

The number of infiltrating TAM, which is evaluated as the number of $\mathrm{CD}^{+} 8^{+}$cells, seems to be inversely correlated with patient prognosis. With the exception of colon cancer and melanoma, the majority of tumors, including breast, kidney, prostate, and uterine corpus, show an increased number of TAM associated with poorer patient prognosis (11). In addition, Takeya's group clearly demonstrated that the number of tumor-infiltrating CD163 and CD204 positive cells, which are other useful markers of TAM, showed an inverse correlation with the prognosis of glioma patients (12). The relationship between TAM infiltration and poor prognosis may in part result from the secretion of epidermal growth factor (EGF) by TAM, which are distributed mainly at the infiltration border of the tumor tissue and perivascular areas at the tumor site. The tumor cells express EGF receptor (EGFR) and are attracted by TAM-secreted EGF, resulting in tumor infiltration into mesenchymal tissue or contact with blood vessels (13-16). Moreover, the fact that TAM infiltration and patient prognosis are not always inversely correlated indicated that various tumors activate TAM differently (11). Our group previously reported that the expression of cytokines, chemokines, and their receptors was tumor-type dependent, but that the site of 
inoculation (skin, liver, or brain in our model) did not affect TAM characteristics (17). Our reports suggest that macrophages can be differentially activated and matured depending on tumor-derived factors.

Plasticity is an important characteristic of macrophages. Indeed, macrophages can be redifferentiated when the microenvironment of cells is changed $(18,19)$. Both monocyte/ macrophages and monocyte-derived dendritic cells (MoDC) originate from a common progenitor, Ly6 $\mathrm{C}^{\text {high }}$ monocyte progenitor cells, and macrophages have the potential to be re-directed toward a MoDC-like phenotype (20). Therefore, in this study, we aimed to redirect TAM toward a dendritic-celllike phenotype utilizing cytokine and siRNA treatment.

\section{Materials and methods}

Mice. Male 6- to 8-week-old C57BL/6 and BALB/c mice were purchased from SLC (Wilmington, MA, USA). OT-II.2a microinjected/Rag1 knockout mice (OT-2 mice) were purchased from Taconic (Germantown, New York, USA).

Cell line. A murine colon carcinoma cell line (MCA38) was cultured in RPMI-1640 medium containing $10 \%$ fetal calf serum, L-glutamine, and penicillin-streptomycin (all from Invitrogen Life Technologies, Tokyo, Japan). Cells were maintained at $37^{\circ} \mathrm{C}$ in a humidified $5 \% \mathrm{CO}_{2}$ atmosphere.

Implantation of tumor cells. Mice were inoculated with $3 \times 10^{6}$ cells injected subcutaneously into the abdominal wall space.

Isolation of tumor-infiltrating cells. Mice were euthanized 14 or 21 days after tumor implantation, and the tumor-infiltrating cells were prepared. The tumors were collected and minced into small pieces before incubation for $15 \mathrm{~min}$ at $37^{\circ} \mathrm{C}$ with the following enzymes dissolved in HBSS: collagenase type I $(0.05 \mathrm{mg} / \mathrm{ml})$, collagenase type IV $(0.05 \mathrm{mg} / \mathrm{ml})$, hyaluronidase $(0.025 \mathrm{mg} / \mathrm{ml}$, all from Sigma Chemical Co., St. Louis, MO, USA), DNase I $(0.01 \mathrm{mg} / \mathrm{ml})$, and soybean trypsin inhibitor (0.2 trypsin inhibitor unit $/ \mathrm{ml}$, both from Roche Diagnostics, Nutley, NJ, USA). Digested cells were harvested, and the red blood cells were lysed with hypotonic buffer $\left(0.155 \mathrm{M} \mathrm{NH}_{4} \mathrm{Cl}\right.$, $0.1 \mathrm{mM}$ EDTA, $10 \mathrm{mM} \mathrm{KHCO}$ ) for $1 \mathrm{~min}$. F $4 / 80^{+}$cells were isolated using biotin-conjugated anti-F4/80 antibody (clone BM8, Invitrogen, Carlsbad, CA), followed by treatment with anti-biotin magnetic immunobeads according to the manufacturer's instructions (MACS, Miltenyi Biotec, BerdishGladbach, Germany).

Multiple PCR analysis. Total RNA was purified using TRIzol (Invitrogen Life Technologies), and 600 ng total RNA was used for reverse transcription with Superscript III reverse transcriptase (Invitrogen Life Technologies). For multiplex PCR analysis, 100 ng cDNA samples or positive control DNA mixtures were mixed with a multiple primer pair mixture, buffer, and Taq polymerase, which were provided by the Multiplex PCR kits for mouse Chemokine Receptors (Sets 1 and 2), Chemokine Genes (Sets 1 and 2), Signaling Receptor (Set 1), Sepsis Cytokines (Set 2), Inflammatory Cytokine Genes (Set 1), CD Antigen (Set 1), and TH1/TH2 Cytokines (Set 2, all from Maxim Biotech, Inc, South San Francisco, CA, USA), and then subjected to PCR using the conditions indicated in the manufacturer's instruction manual. The amplified DNAs were analyzed by $5 \%$ acrylamide gel electrophoresis followed by ethidium bromide staining.

Real-time PCR analysis. Total RNA was purified using TRIzol (Invitrogen Life Technologies), and $600 \mathrm{ng}$ total RNA was used for reverse transcription with Superscript III reverse transcriptase. For real-time PCR analysis, $5 \mu \mathrm{l}$ cDNA samples diluted 20X, $1 \mu \mathrm{l}$ each of the upper and lower primer, $3 \mu 1$ PCR-grade water (Roche Diagnostics, Indianapolis, IN, USA), and $10 \mu 12 \mathrm{X}$ concentrated SYBR Green and Taq enzymepremixed reaction mixture $\left(\mathrm{SYBR}^{\circledR}\right.$ Premix Ex Taq ${ }^{\mathrm{TM}}$, Takara Bio, Japan) were used. Some of the primer pair sequences used in this analysis are indicated in our previous papers $(21,22)$. The sequences of the primer pairs used only in this report and not previously are: IFN $\gamma, 5$ '-CGGGAC AGTCATTGAAAGCCTA-3', 5'-GTTGCTGATGGCCTG ATTGTC-3'; CCR2, 5'-GCAAGTTCAGCTGCCTGCAA-3', 5'-ATGCCGTGGATGAACTGAGGTAA-3'; CX3CR1, 5'-TGACCCTGCAAGCATCACGTA-3', 5'-CAATGTAA GCCTGCAAATGAGACC-3'; IL-4, 5'-TCTCGAATGTAC CAGGAGCCATATC-3', 5'-AGCACCTTGGAAGCCCTAC AGA-3'; IL-6, 5'-CCACTTCACAAGTCGGAGGCTTA-3', 5'-GCAAGTGCATCATCGTTGTTCATAC-3'. The reaction conditions consisted of one 5 -min cycle at $95^{\circ} \mathrm{C}$, followed by 45 cycles at $95^{\circ} \mathrm{C}$ for $10 \mathrm{sec}$, and $72^{\circ} \mathrm{C}$ for $10 \mathrm{sec}$. The reaction and analysis were performed with a Light Cycler instrument (Roche Diagnostics, Indianapolis, IN, USA).

SDS-PAGE and immunoblotting. $\mathrm{F} 4 / 80^{+}$cells isolated from tumor tissues were washed three times with PBS before being incubated on ice for $1 \mathrm{~h}$ with lysis solution [50 mM Tris-HCl, pH. 7.5, $150 \mathrm{mM} \mathrm{NaCl}, 1 \%$ Triton X-100, 1 mM EDTA, $1 \mathrm{mM}$ EGTA, $1 \mathrm{mM}$ PMSF, $5 \mathrm{mM}$ iodoacetamide, $1 \mathrm{mM} \mathrm{Na}_{3} \mathrm{VO}_{4}$, and protease inhibitor cocktail (Sigma Chemical Co., P-8340)]. The supernatants were collected and subjected to electrophoresis on $10 \%$ PAGE gels. After transferring the proteins onto polyvinylidene difluoride membranes (Bio-Rad Laboratories, Hercules, CA, USA), the membranes were blocked with skim milk and then reacted for $1 \mathrm{~h}$ with $2 \mu \mathrm{g} / \mathrm{ml}$ of the following antibodies diluted in a primary antibody signalenhancing solution (Can Get Signal ${ }^{\mathrm{TM}}$, Immunoreaction Enhancer Solution 1, Toyobo Co, Ltd., Osaka, Japan): antimouse stat 1 (C-terminus, clone 42), anti-mouse phospho-specific stat1 (pY701, clone 14), anti-mouse stat3 (clone 84), anti-mouse phospho-specific stat3 (pY705, clone 4), anti-mouse stat4 (clone 8), anti-mouse phospho-specific stat4 (pY693, clone 38, all from BD Transduction Laboratories), anti-mouse stat5 (rabbit polyclonal), anti-mouse phospho-specific stat5 (Tyr694, rabbit polyclonal), anti-stat6 (rabbit polyclonal, all from Cell Signaling), anti-mouse phospho-specific stat6 (Y641, clone 16E12, Millipore Corporation), anti-mouse NF- $\mathrm{\kappa Bp} 65$ (rabbit polyclonal, Cell Signaling), anti-mouse NF- $\mathrm{kBp} 105 / \mathrm{p} 50$ (clone 12A2F, Abcam), anti-mouse ERK (panERK, clone 16), antimouse ERK1 (clone MK12), anti-mouse pan-JNK/SAPK1 (clone 37), anti-mouse arginase I (clone 19), anti-mouse iNOS (clone 2), anti-mouse iNOS/NOS type II (clone 6, all from BD Transduction Laboratories). After washing, the membranes were stained with $16 \mathrm{ng} / \mathrm{ml} \mathrm{HRP}$-conjugated goat anti-rabbit 

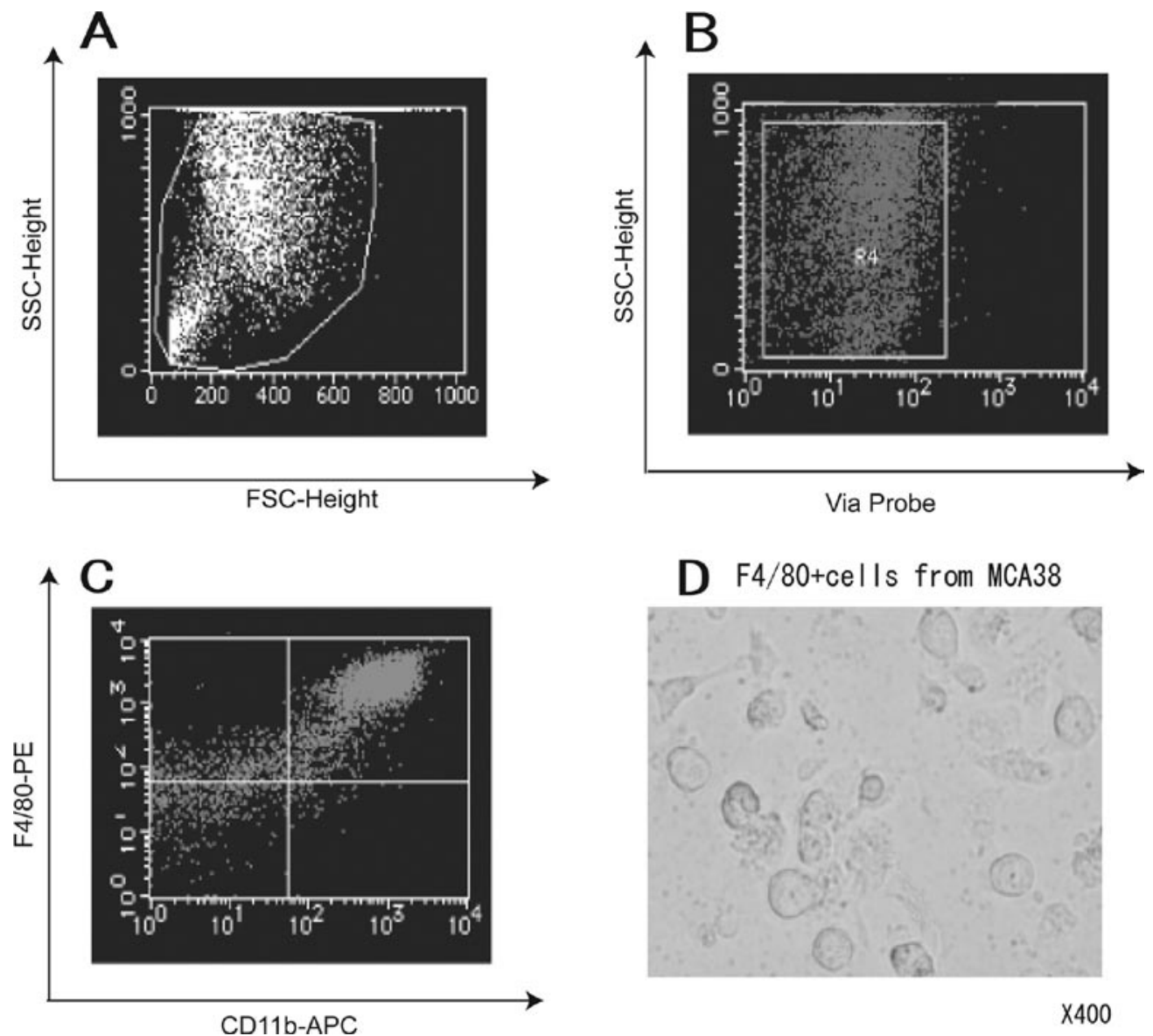

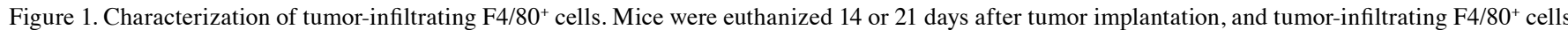
were purified using an F4/80 positive immunobeading method (A-C). Dead cells were excluded by Via-Probe (B), and the CD11b and F4/80 2-D histogram is shown (C). Appearance of $\mathrm{F} 4 / 80^{+}$cells after $24 \mathrm{~h}$ cell culture with medium is shown. Almost all cells adhered to the dish and demonstrated a short spindle shape (D). Representative data from three independent experiments are shown.

IgG or anti-mouse IgG (both from Jackson ImmunoResearch Laboratories, Inc., West Grove, PA, USA) diluted in Can Get Signal $^{\mathrm{TM}}$ Immunoreaction Enhancer Solution 2 (Toyobo Co., Ltd.) for $1 \mathrm{~h}$. The blots were developed with ECL (Amersham Pharmacia Biotech, Buckinghamshire, UK), and the images were captured by the Cool Saver Lumino capture system (Model AE-6955, ATTO, Tokyo, Japan) before analysis using CS Analyzer software (ATTO). The membrane was then stripped and reprocessed with $2 \mu \mathrm{g} / \mathrm{ml}$ anti-GAPDH (clone 9.B.88, United States Biological, Swampscott, MA, USA), and $16 \mathrm{ng} / \mathrm{ml}$ HRP-conjugated goat anti-mouse IgG (Jackson ImmunoResearch Laboratories, Inc.).

siRNA silencing. Cells were transfected with $0.4 \mathrm{nmol} / \mathrm{ml}$ siRNA using Lipofectamine 2000 (Invitrogen Life Technologies) according to the manufacturer's instructions. The following siRNA sequences, designed by the manufacturer (Invitrogen, Carlsbad, CA), were used: M-CSFR1, 5'-UAGCCUUGCGG AUAAUGAACCCUCG-3' and 5'-CGAGGGUUCAUUAU CCGCAAGGCUA-3'; control siRNA, 5'-UAGCCCGUUCA GGCUAAUCAAGUCG-3' and 5'-CGACUUGAUUAGCC UGAACGGGCUA-3'.

Mitomycin $C$ treatment of splenocytes. Splenocytes were prepared from male 6 - to 8 -week-old BALB/c mice. The collected splenocytes were treated with hypotonic buffer $\left(0.155 \mathrm{M} \mathrm{NH}_{4} \mathrm{Cl}, 0.1 \mathrm{mM}\right.$ EDTA, $10 \mathrm{mM} \mathrm{KHCO}_{3}$ ) for $1 \mathrm{~min}$ to lyse red blood cells. The cells were then treated with $100 \mu \mathrm{g} / \mathrm{ml}$ mitomycin C (MMC) in RPMI-1640 medium for $30 \mathrm{~min}$ at $37^{\circ} \mathrm{C}$. After washing with PBS three times, the cells were used as allostimulator cells in mixed lymphocyte culture.

Analysis of antigen presentation by T-cell proliferation. Alloantigen stimulation and ovalbumin antigen-specific T-cell systems were utilized. For the alloantigen stimulation system, responder cells were prepared as the negative population of magnetic immunobeading (Miltenyi Biotec, Germany) with anti-CD11b, CD11c, and B220 immunobeads. Responder cells were labeled with CFSE (Invitrogen) according to manufacturer's instructions for flow cytometric analysis. One million responder cells, $1 \times 10^{6}$ allostimulator cells, and $1 \times 10^{3}$ antigenpresenting cells $\left(\mathrm{F} 4 / 80^{+}\right.$cells treated with either control or M-CSFR1 siRNA for 5 days) were co-cultured in one well of a 24-well plate containing $2 \mathrm{ml}$ medium for 5 days and then collected for analyses. For the ovalbumin-specific T-cell system, ovalbumin (OVA) was utilized instead of allostimulator cells, and OT-2 mouse-derived splenocytes were prepared by the same method used for responder-cell preparation in the alloantigen system. OVA $(1 \mathrm{mg} / \mathrm{ml}), 1 \times 10^{6}$ CFSE-labeled responder cells, and $1 \times 10^{3}$ antigen presenting cells (F4/80 ${ }^{+}$cells 

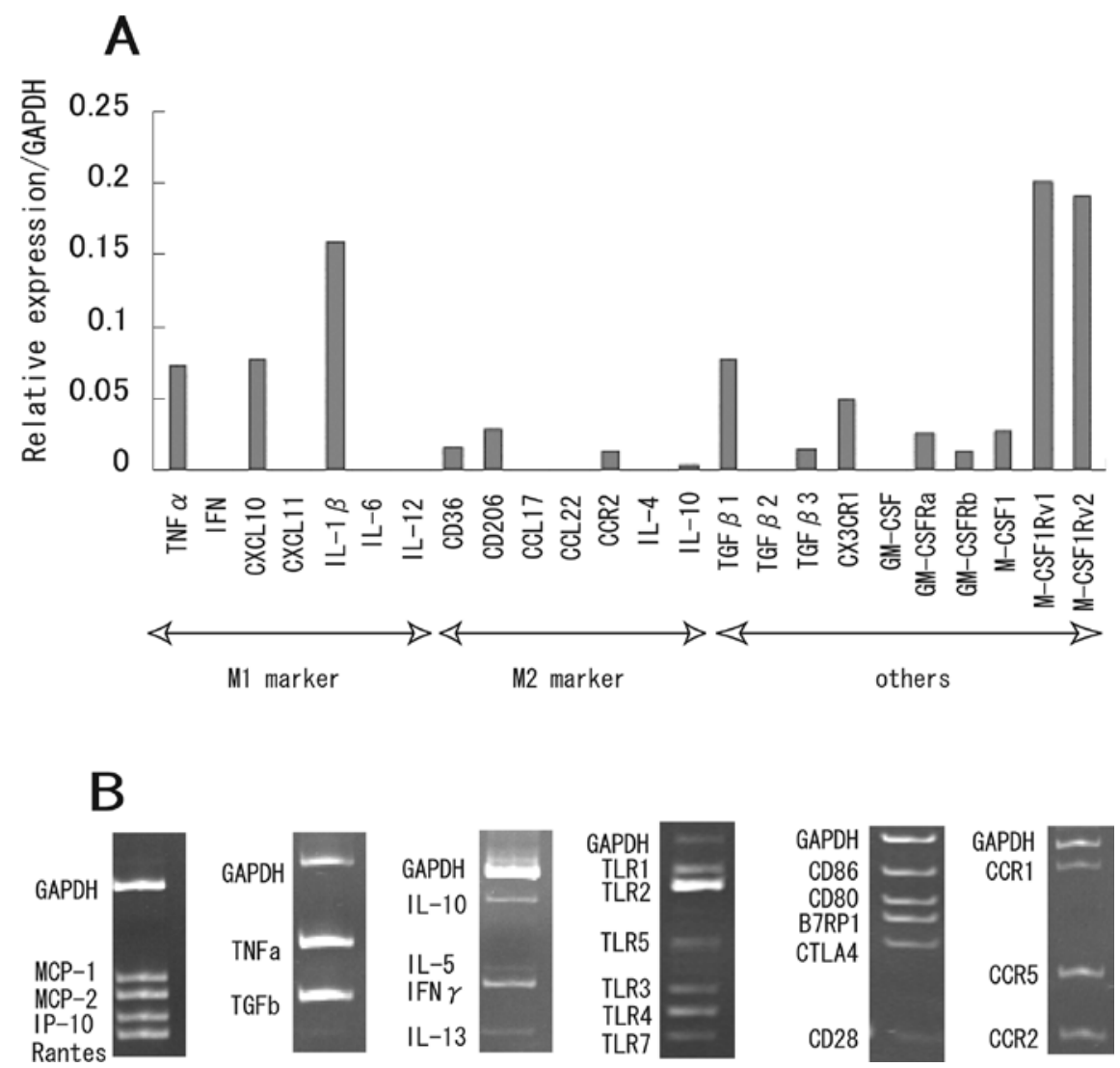

Figure 2. Characterization of freshly-isolated tumor-associated macrophages (F4/80+ cells) based on M1/M2 classification. Real-time PCR analysis of TAM for the expression of cytokines, chemokines, and their receptors based on M1/M2 classification is shown as the relative expression level to GAPDH (A). The same cells were investigated with multiple PCR analysis (B). Representative data from two independent experiments are shown.

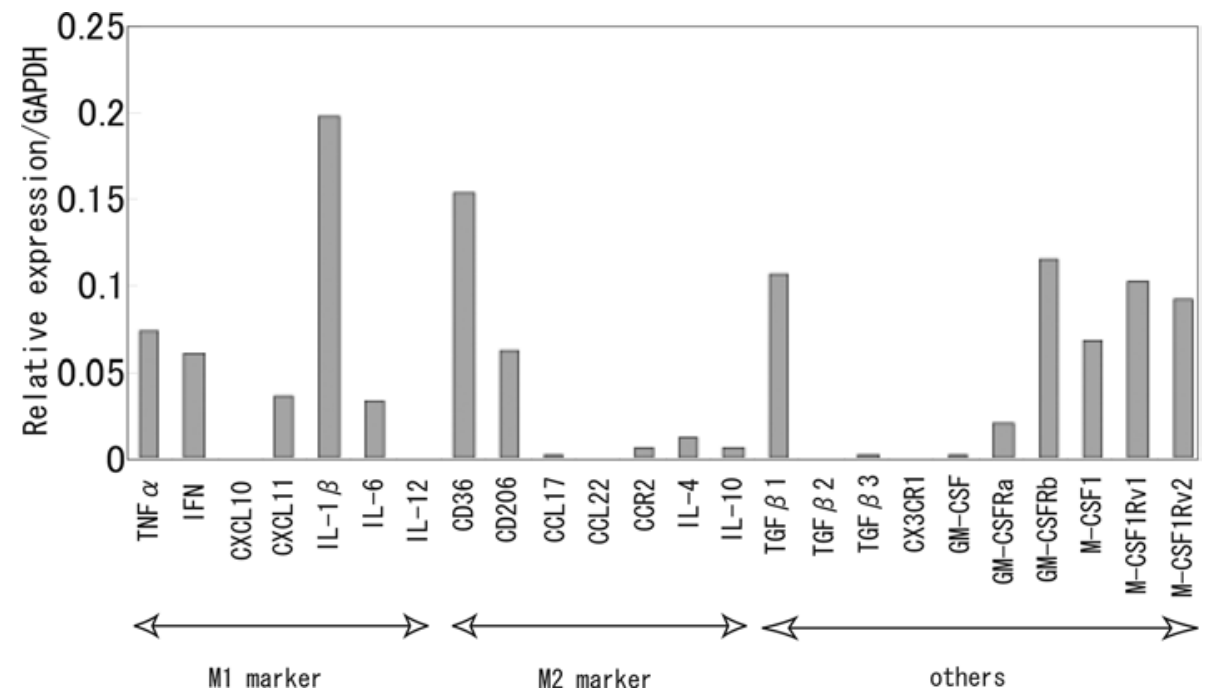

Figure 3. Effect of GM-CSF treatment on macrophage differentiation based on the M1/M2 classification of tumor-associated macrophages using real-time PCR. TAM were cultured with GM-CSF for 3 days. Real-time PCR analysis for the expression of cytokines, chemokines, and their receptors is shown relative to the expression level of GAPDH. Representative data from three independent experiments are shown.

treated with either control or M-CSFR1 siRNA for 5 days) were co-cultured in one well of a 24-well plate containing $2 \mathrm{ml}$ medium for 5 days and then collected for analyses.

For flow cytometric analysis, the cells were preincubated with $10 \mu \mathrm{g} / \mathrm{ml}$ anti-CD16/32 (clone 2.4G2, BD Biosciences,
Rockville, MD, USA) at $4^{\circ} \mathrm{C}$ for $30 \mathrm{~min}$ prior to staining. Via-Probe (BD Biosciences) was used for dead cell exclusion according to the manufacture's instructions. The following antibodies were used at $10 \mu \mathrm{g} / \mathrm{ml}$ : APC-conjugated anti-CD3 (clone 145C11), PE-conjugated anti-CD4 (RM4-5), and 
$\mathrm{F} 4 / 80+$ cells

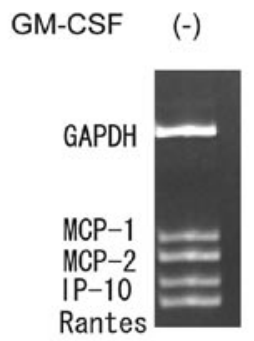

$(+)$

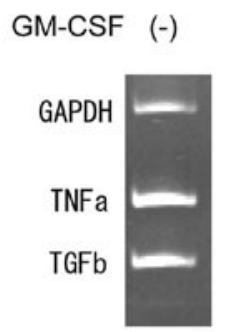

$\mathrm{F} 4 / 80+$ cells

$(+)$

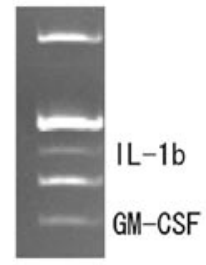

$\mathrm{F} 4 / 80+$ cells

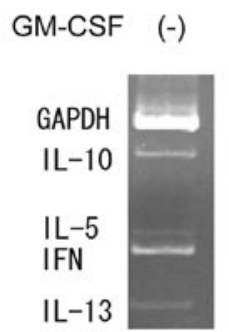

$(+)$

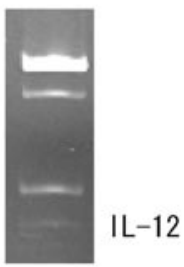

$\mathrm{F} 4 / 80+$ cells

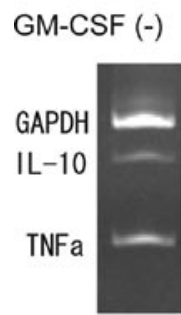

$(+)$

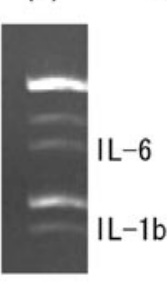

GM-CSF

$\mathrm{F} 4 / 80+$ cells

$(-)$

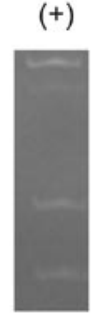

$\mathrm{F} 4 / 80+$ cells

GM-CSF (-)
GAPDH
TLR1
TLR2
TLR5
TLR3
TLR4
TLR7

(+) GM-CSF (-)

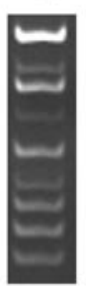

$\mathrm{F} 4 / 80+$ cells

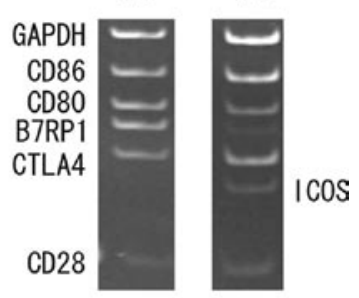

B

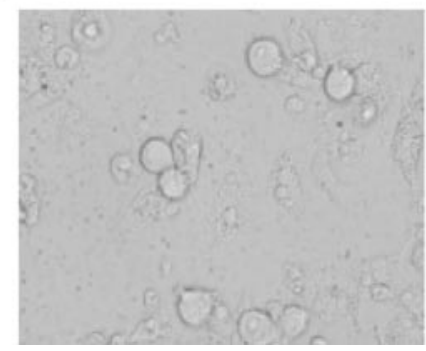

Figure 4. Effect of GM-CSF treatment on macrophage differentiation based on the M1/M2 classification of tumor-associated macrophages using multiple PCR analysis. F4/80 $0^{+}$cells were cultured with or without GM-CSF for 3 days. Multiple PCR analysis for the expression of cytokines, chemokines, and their receptors was performed (A). Appearance of TAM after 3 days of in vitro culture with GM-CSF (B). Representative data from three independent experiments are shown.
PE-conjugated anti-CD8b (clone 53-5.8, BD Biosciences). The cells were incubated with the antibodies for $30 \mathrm{~min}$ at $4{ }^{\circ} \mathrm{C}$ and washed with PBS. The samples were analyzed by a FACS Calibur flow cytometer and CellQuest software (BectonDickinson Japan, Tokyo). For cell proliferation assays, co-cultured cells were mixed well, distributed into a 96-well plate $(180 \mu \mathrm{l}$ cells/well), and $20 \mu \mathrm{l}$ CellTiter 96 reagent (Promega Corp, Madison, WI, USA) was added to each well. The cells were cultured for $1 \mathrm{~h}$ at $37^{\circ} \mathrm{C}$, and the reaction was then measured by a microplate reader (Bio-Rad Laboratories) at $490 \mathrm{~nm}$ and analyzed using MPM-3 Microplate Manager software (Bio-Rad Laboratories).

Statistical analysis. The Student's-t test was used for statistical analysis. $\mathrm{P}<0.05$ was considered as a significant difference.

\section{Results}

Tumor-associated macrophages bear characteristics of both M1- and M2-type macrophages. To characterize purified tumor-infiltrating $\mathrm{F} 4 / 80^{+}$cells, mice were euthanized 14 or 21 days after tumor implantation, and tumor-infiltrating $\mathrm{F} 4 / 80^{+}$cells were isolated. Purified cells were almost entirely
F4/80 ${ }^{\text {high }}$ and $C D 11 b^{\text {high }}$, which indicated that the population was suitable for consideration as tumor-associated macrophages (TAM) (Fig. 1). Macrophages were classified using the M1/M2 scheme (23) based on their expression pattern of M1/ M2 factors using real-time PCR analysis. As shown in Fig. 2, TAM expressed not only M2-type markers such as CCL22, CCL17, IL-10, and CD206, but also M1-type markers, including IL-1 $\beta$, TNF $\alpha$, and CXCL10. The data indicated that the TAM utilized in this study simultaneously expressed both M1 and M2 factors.

Tumor-associated macrophages do not change character significantly when cultured with GM-CSF. Because macrophages have been reported to demonstrate plasticity, we investigated whether TAM can be redirected to dendritic cells using in vitro GM-CSF treatment because GM-CSF has been used previously to investigate DC differentiation by monocytes/ macrophages (19). TAM were cultured with or without GMCSF for 3 days (Fig. 3). The expression levels of M1-type markers (including IL-1 $\beta, \mathrm{TNF} \alpha$, and CXCL10) decreased, but the levels of M2-type markers (including CD36 and CD206) were unchanged. The expression levels of CCR1, CCR2, CCR5, and IL-13 decreased, IL-1 $\beta$ was unchanged, and IL- 6 and 

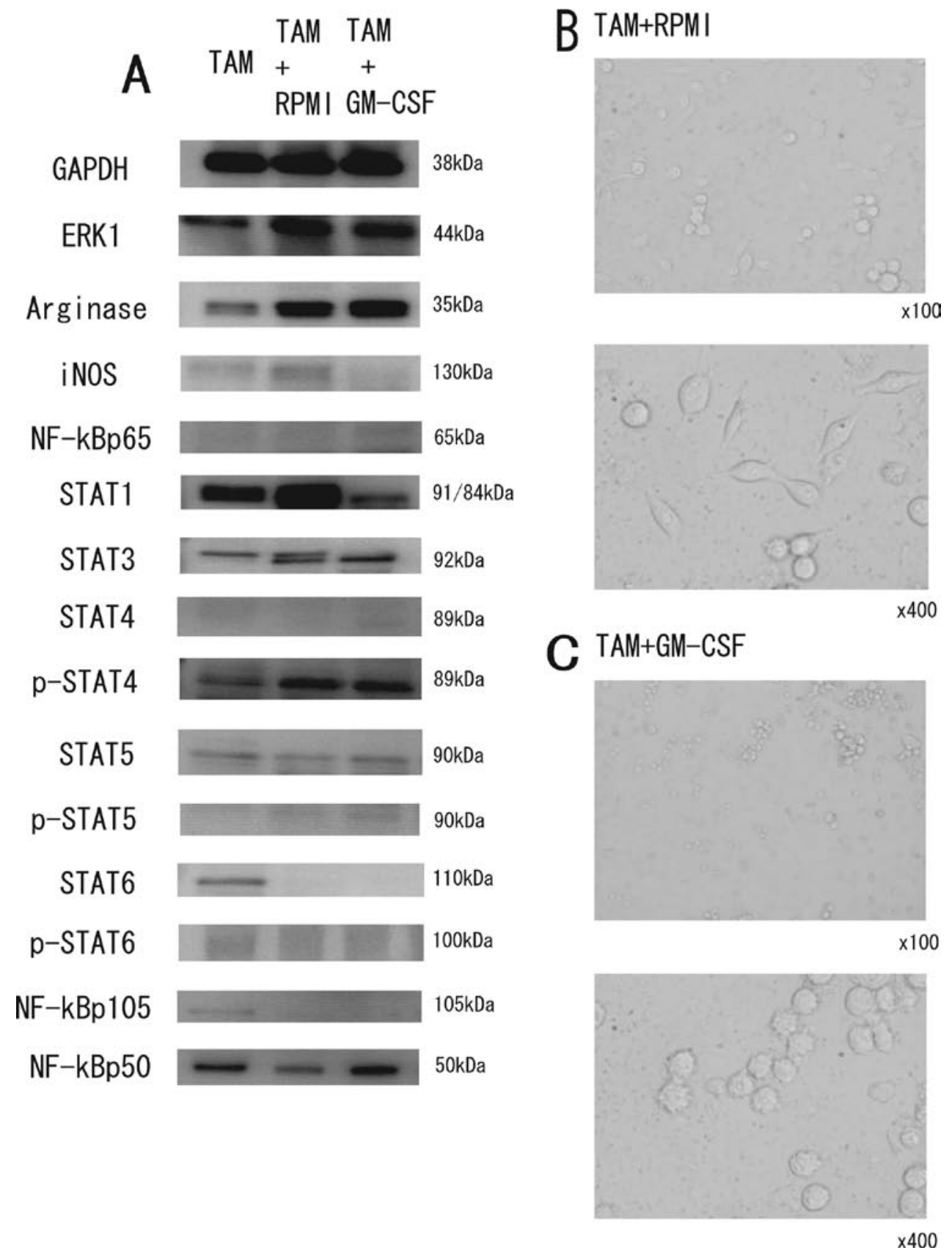

Figure 5. Tumor-associated macrophages (F4/80 $0^{+}$cells) were cultured for 5 days in RPMI medium with or without GM-CSF. Immunoblot analysis of intracellular signaling pathways (A). Appearance of TAM from MCA38 tumors after in vitro culture without GM-CSF (B) and with GM-CSF (C). Representative data from three independent experiments are shown.

IL-12 were only detected after GM-CSF treatment (Fig. 4). When the expression of intracellular signal transduction proteins was compared between freshly isolated TAM and TAM cultured for 5 days with or without GM-CSF treatment (Fig. 5A), a few differed across groups (including STAT1, p-STAT5, STAT6, NF-кB), but others did not. In terms of morphology, GM-CSF treatment apparently decreased the adhesive ability of TAM; the cells were round and floated in the medium (Fig. 5B and C).

Blocking the tumor-associated macrophage M-CSF pathway in the presence of GM-CSF leads to altered intracellular signal transduction. Because the importance of M-CSF in macro- phage activation has been demonstrated (24), the combined effect of M-CSF signal blockade and GM-CSF treatment on TAM was then investigated in vitro. No significant differences were observed in the expression pattern of M1/M2 factors analyzed using multiple PCR (Fig. 6). In terms of TAM intracellular signal transduction (Fig. 7A), the expression levels of all molecules were unchanged in TAM treated with control siRNA and GM-CSF compared to TAM cultured with GMCSF alone, as shown in Fig. 5A. In contrast, M-CSFR knockdown by siRNA for M-CSFR1 led to increased expression levels of STAT5, STAT6, and NF-кBp65 (Fig. 7A). The morphology of cells treated with control siRNA and GM-CSF was similar to that of cells treated with GM-CSF alone (Figs. 5C 

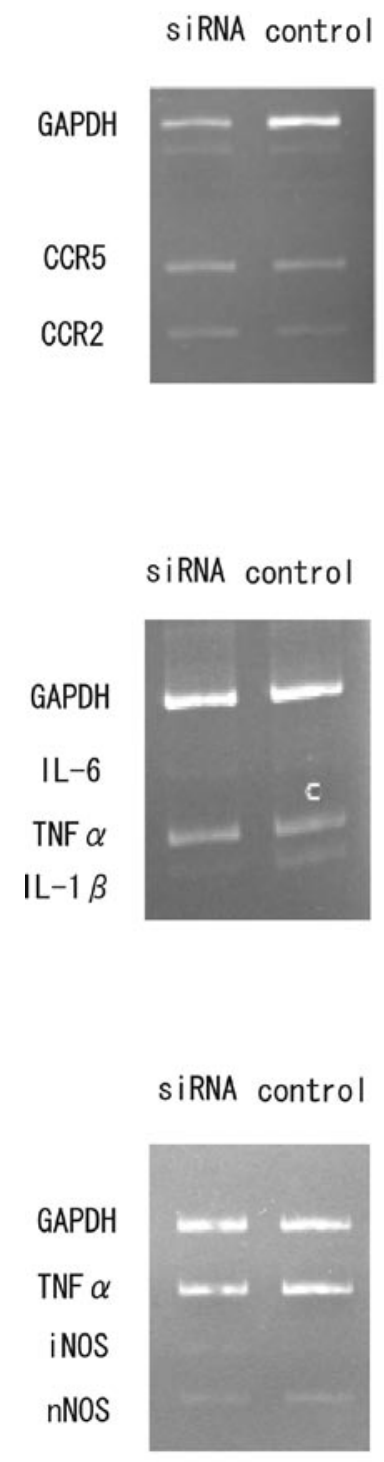

siRNA control

GAPDH

CX3CR1

CCR9

CCR7

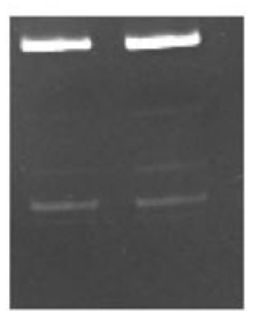

siRNA control

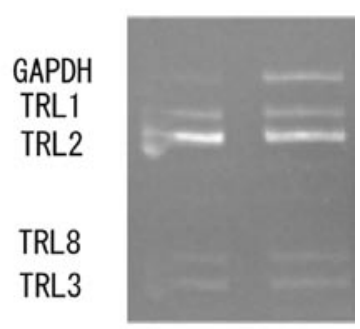

siRNA control

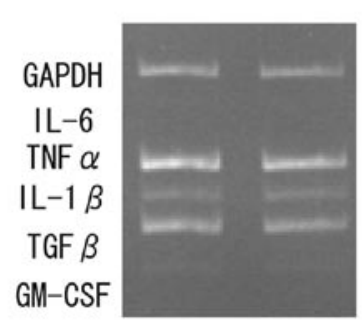

siRNA control

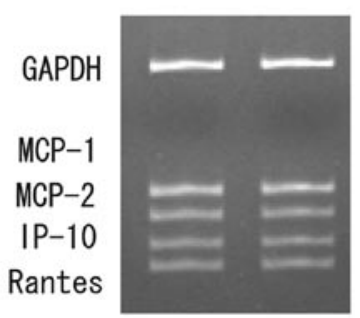

siRNA control

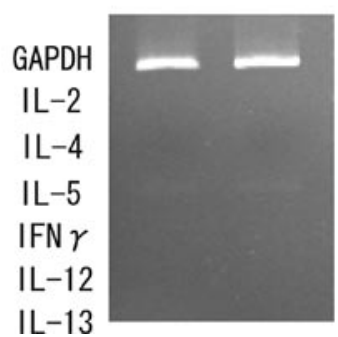

Figure 6. TAM M-CSFR knockdown by M-CSFR1 and control siRNA. Multiple PCR analysis of the expression pattern of M1/M2 factors. Representative data from three independent experiments are shown.

and 7B). However, M-CSFR knockdown by M-CSFR1 siRNA led to a change in cell morphology to a dendritic shape (Fig. 7C).

Blockade of the M-CSF pathway in conjunction with GM-CSF treatment sustains the antigen-presentation capability of tumor-associated macrophages. Finally, the antigenpresentation capability of TAM was examined. T cells were stimulated, but proliferation did not differ between control and M-CSFR1 siRNA conditions (both with GM-CSF treatment), as analyzed by two different antigen-responder combinations (alloantigen and ovalbumin systems) (Fig. 8).

\section{Discussion}

Macrophages have been classified into two subtypes based on the concept of Th1/2 T-cell subtype classification, because macrophage differentiation is strongly influenced by IFN $\gamma$ and IL-4, which are secreted by Th1- and Th2-type T-cells, respectively (25). Based on this classification, TAM have been reported to fall into the M2 subtype (23). In this study, TAM (tumor-infiltrating F4/80+ cells) were first assessed for their expression of cytokines, chemokines, membrane proteins, and intracytoplasmic signaling molecules related to macrophage activation and maturation. M1-type (IL-1 $\beta$, TNF $\alpha$, CXCL-10) and M2-type macrophage markers (CD206, CD36, CCL17) were found to be simultaneously expressed by TAM, indicating that our tumor-infiltrating $\mathrm{F} 4 / 80^{+}$cells had the unique characteristic of expressing both M1 and M2 molecules (Figs. 1 and 2). These results are almost identical to previously reported findings that utilized tumor-infiltrating macrophages prepared by CD11b-positive immunobeading $(22,26)$.

We previously reported that TAM strongly secrete TNF $\alpha$, IL-1 $\beta$, and TGF $\beta$, and that inhibition of TGF $\beta$ blocked the immunosuppressive characteristics of TAM (22). We also reported that an immunogene therapy model utilizing IL-2 and STNFR2 in combination suppressed TAM differentiation (21). These data suggested that macrophages have plasticity, and that different tumor microenvironments can differentially activate and differentiate macrophages to unique tumor-based 

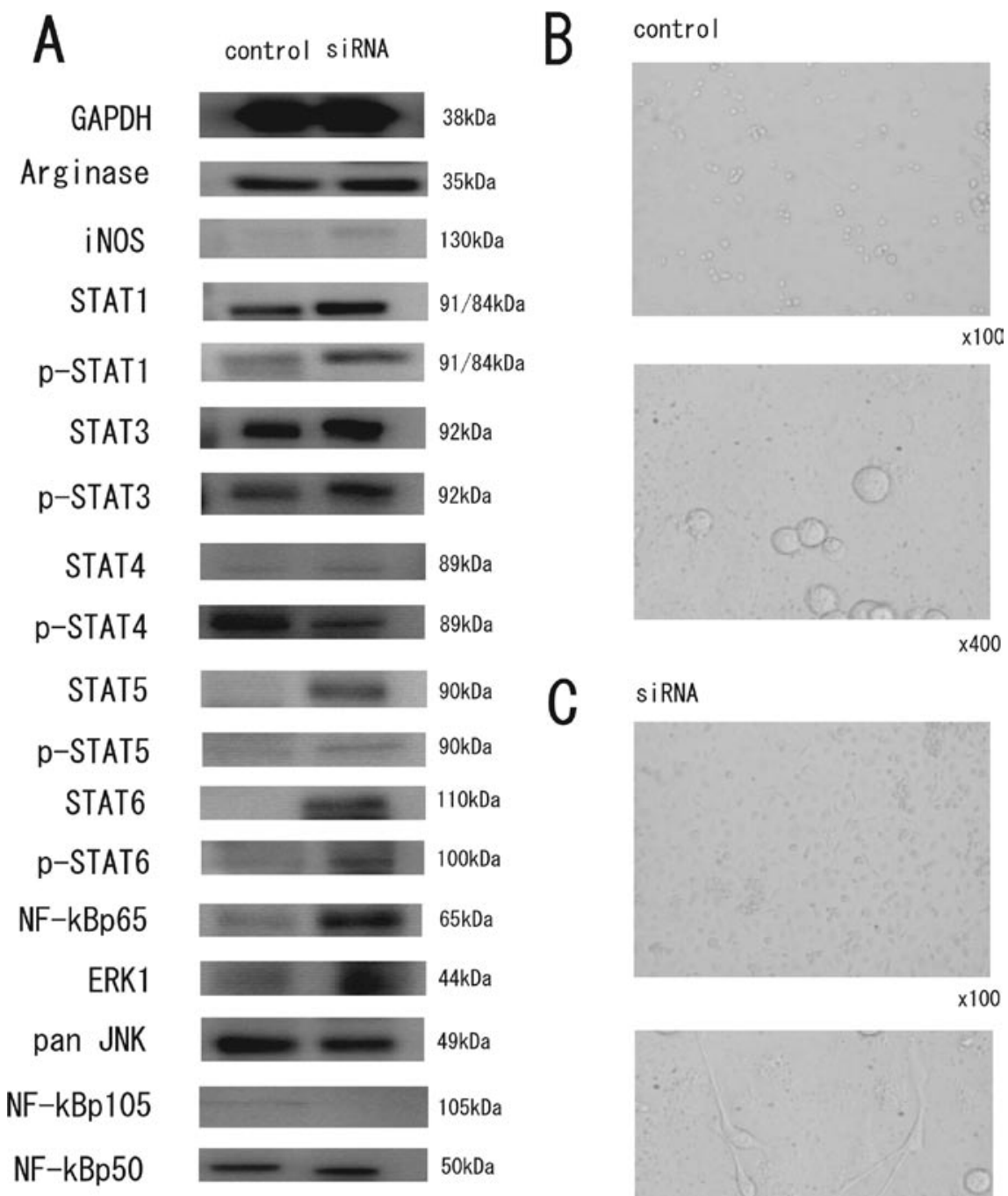

C
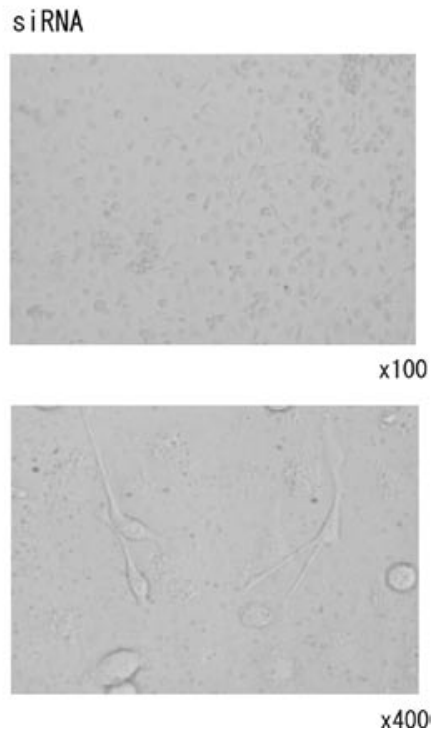

Figure 7. TAM M-CSFR knockdown by M-CSFR1 and control siRNA. Immunoblotting analysis of intracellular signal transduction (A). Appearance of TAM cultured with GM-CSF in RPMI medium (B). Appearance of TAM treated with siRNA (M-CSFR) in the presence of GM-CSF (C). Representative data from three independent experiments are shown.

phenotypes. This finding could be explained by the plasticity of macrophages for reprogramming as stated also previously $(18,19,27)$. Therefore, in this study, we aimed to investigate the possibility that TAM could be redirected toward a dendritic cell-like phenotype by GM-CSF treatment in vitro, because GM-CSF was reported to be a strong monocyte differentiation factor for dendritic cells $(28,29)$.

Contrary to our prediction, incubation of TAM with GM$\mathrm{CSF}$ in vitro did not change the expression of M1/M2 molecules as determined and shown in Figs. 1 and 2 (Figs. 3, 4 and 5A), although the morphology of TAM changed to round cells that showed less adhesion to the culture dish (Fig. 5B and C). This could have been because TAM were more mature than monocytes previously differentiated by GM-CSF alone in vitro $(28,30)$, suggesting that several factors were necessary for the redifferentiation of TAM. A single administration GM-CSF has also been shown not to be fully successful in tumor immunotherapy $(31,32)$, which is supported by our present in vitro results concerning GM-CSF treatment alone. Therefore, in the case of GM-CSF tumor immunotherapy in vivo, TAM may remain at the tumor site and retain their immunosuppressive properties. Subsequently, we attempted to suppress M-CSF signaling, because M-CSF is essential for monocyte differentiation into macrophages $(33,34)$. Indeed, several reports suggest that blocking M-CSF signaling by M-CSFR knock out, anti-M-CSF antibody, or anti-M-CSFR antibody can change the function or morphology of macrophages and induce dendritic cell-like characteristics (35-38). Therefore, in this study, M-CSFR siRNA was used in the presence of GM-CSF, with the expectation that M-CSF signal blockade could suppress TAM maturation, while GM-CSF could redirect immature TAM toward a dendritic cell-like phenotype.

Interestingly, combination treatment of TAM with GM-CSF and M-CSFR siRNA affected the signal transduction pathway of TAM but not the expression pattern of M1/M2 marker molecules (Fig. 7). A number of previous studies have investi- 

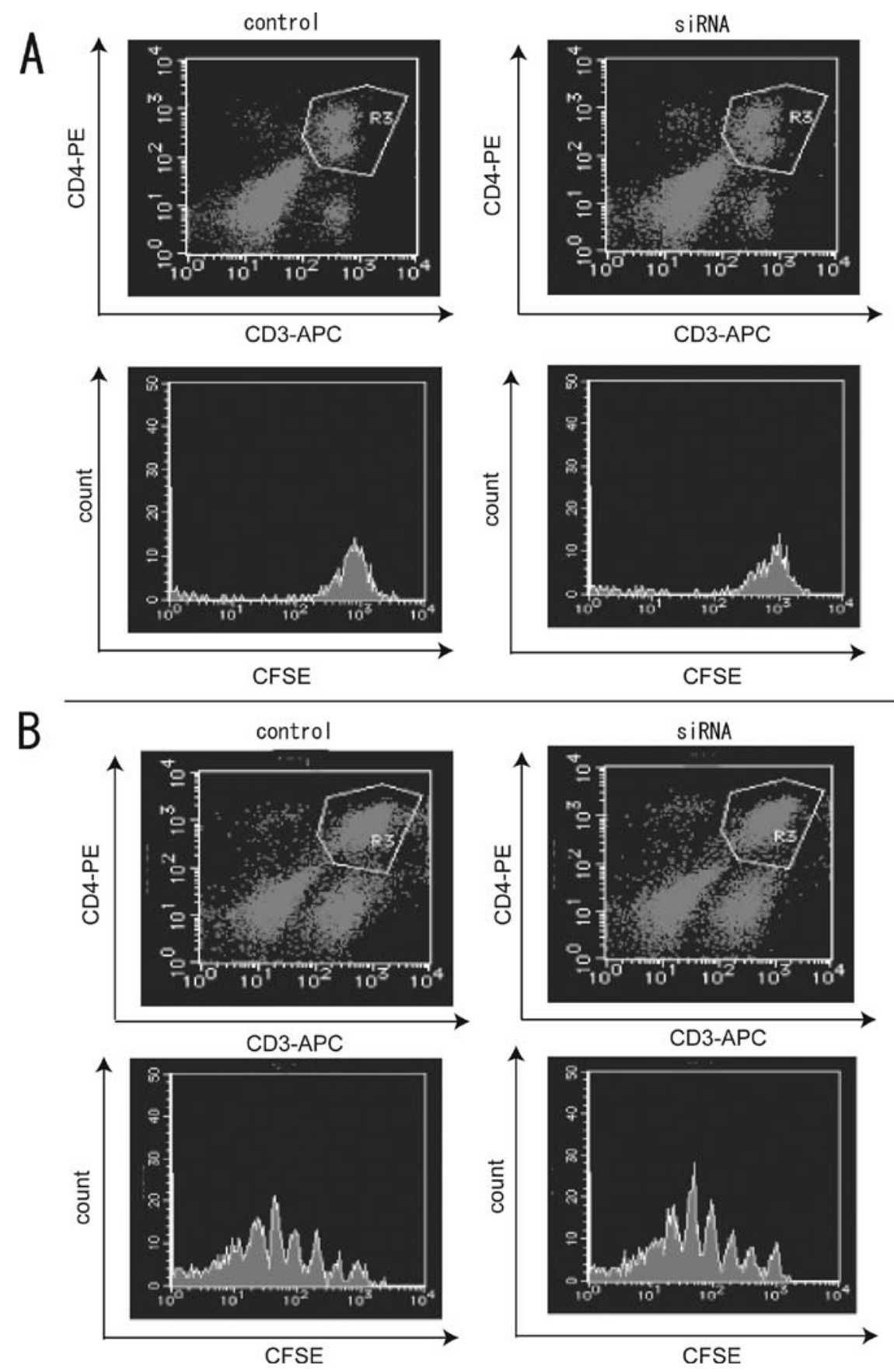

Figure 8. Analysis of the antigen presenting capability of TAM. Antigen responder T cells derived from BALB/c and OT-2 mouse splenocytes were labeled with CFSE. $C D 4^{+} \mathrm{T}$ cells among responder cells were analyzed by flow cytometry after stimulation with alloantigen (A) or ovalbumin antigen-specific systems (B). Representative data from two independent experiments are shown.

gated the STAT pathway in monocytes and dendritic cells. STAT5 was not induced in mature dendritic cells by GM-CSF treatment alone (39), although the same treatment of monocytes induced STAT5 and monocyte differentiation into dendritic cells (40). STAT6 was expressed by immature dendritic cells and was induced by treating monocytes with IL-4, which resulted in dendritic cell maturation (40). In contrast, GM-CSF treatment of macrophages did not induce STAT6 expression, but IL-4 treatment of the same cells did induce STAT6 (41). The IL-6/STAT3 pathway was essential for the redirection of monocytes from dendritic cell differentiation toward macro- phage differentiation, and STAT3 was essential for macrophage survival (42). In terms of TAM, STAT3 did not induce, but spleen monocytes of tumor-bearing animals expressed STAT3 (43). STAT1 was consistently expressed at all steps of dendritic cell differentiation and was essential for the maturation of monocytes into dendritic cells (40). While STAT1 was expressed by TAM, monocytes from the spleens of tumor-bearing animals did not express STAT1 (43).

Previous findings and our present results indicate that treatment of tumor-infiltrating $\mathrm{F} 4 / 80^{+}$macrophages with M-CSFR siRNA in combination with GM-CSF induced 
STAT1, 5, and 6, all of which are essential for dendritic-cell maturation (40). Previous studies have shown that, in terms of the NF- $\mathrm{kB}$ pathway, p50 but not p65 are usually expressed by TAM (44-47). As shown in Fig. 7, GM-CSF and M-CSFR siRNA combination treatment significantly induced $\mathrm{p} 65$ expression, while p50 and p105 expression levels were not affected by the treatment. These results indicated that the combination of GM-CSF and M-CSFR siRNA could induce a different cell signaling pathway from that originally utilized by TAM. GM-CSF and M-CSFR siRNA treatment did not affect the capacity for antigen presentation (Fig. 8). This finding is understandable given that activated macrophages already have the ability for antigen presentation $(48,49)$.

Taken together, our present data indicate that our treatment model could not redirect TAM to a MoDC-like phenotype based on the analysis of M1/M2 marker expression (Fig. 6), but could direct cell signaling pathways toward a dendritic cell-like pattern. Thus, treatment of tumor-bearing hosts by factors, such as GM-CSF, and blockade of M-CSF, as utilized in this study, could affect TAM differentiation via the plasticity of macrophages. Indeed, blockade of the NF- $\mathrm{KB}$ pathway has been shown to redirect TAM from an immunosuppressive phenotype to one with tumor-killing activity (M1 type) (27).

Tumor immunotherapy is a useful therapeutic strategy because it has fewer adverse effects than chemotherapy (50). However, because immune mechanisms are complicated, the effects of immunotherapeutic drugs have not been fully understood. Controlling the maturation and differentiation of tumor-infiltrating immune cells is critical for the development of effective immunotherapies. Macrophages remain at the sites of inflammation (such as vascular wall and soft tissue), while neutrophils die after degranulation (51). Considering that TAM are immunosuppressive $(10,52)$ and remain at the tumor site due to their macrophage characteristics, suppressing the function and maturation of TAM, in addition to considering how to elicit anti-tumor immune responses, is very important.

\section{Acknowledgements}

This study was supported in part by a Grant-in-Aid for Scientific Research (Grants 21590415, and 21590416) from the Japan Society for the Promotion of Science.

\section{References}

1. Gabrilovich DI, Bronte V, Chen SH, et al: The terminology issue for myeloid-derived suppressor cells. Cancer Res 67: 425-426, 2007.

2. Young MR, Newby $M$ and Wepsic HT: Hematopoiesis and suppressor bone marrow cells in mice bearing large metastatic Lewis lung carcinoma tumors. Cancer Res 47: 100-105, 1987.

3. Bronte V, Serafini P, Apolloni E, et al: Tumor-induced immune dysfunctions caused by myeloid suppressor cells. J Immunother 24: 431-446, 2001, 1997.

4. Gallina G, Dolcetti L, Serafini P, et al: Tumors induce a subset of inflammatory monocytes with immunosuppressive activity on CD8 ${ }^{+}$T cells. J Clin Invest 116: 2777-2790, 2006.

5. Bronte V, Apolloni E, Cabrelle A, et al: Identification of a $\mathrm{CD} 11 \mathrm{~b}(+) / \mathrm{Gr}-1(+) / \mathrm{CD} 31(+)$ myeloid progenitor capable of activating or suppressing CD8(+) T cells. Blood 96: 3838-3846, 2000.

6. Young MR and Lathers DM: Myeloid progenitor cells mediate immune suppression in patients with head and neck cancers. Int J Immunopharmacol 21: 241-252, 1999.
7. Young MR, Aquino S and Young ME: Differential induction of hematopoiesis and immune suppressor cells in the bone marrow versus in the spleen by Lewis lung carcinoma variants. J Leukoc Biol 45: 262-273, 1989.

8. Young MR, Young ME and Wright MA: Myelopoiesis-associated suppressor-cell activity in mice with Lewis lung carcinoma tumors: interferon-gamma plus tumor necrosis factor-alpha synergistically reduce suppressor cell activity. Int J Cancer 46: 245-250, 1990

9. Rabinovich GA, Gabrilovich D and Sotomayor EM: Immunosuppressive strategies that are mediated by tumor cells. Annu Rev Immunol 25: 267-296, 2007.

10. Mantovani A, Bottazzi B, Colotta F, et al: The origin and function of tumor-associated macrophages. Immunol Today 13: 265-270, 1992.

11. Lewis CE and Pollard JW: Distinct role of macrophages in different tumor microenvironments. Cancer Res 66: 605-612, 2006.

12. Komohara Y, Ohnishi K, Kuratsu J, et al: Possible involvement of the M2 anti-inflammatory macrophage phenotype in growth of human gliomas. J Pathol 216: 15-24, 2008.

13. Sahai E, Wyckoff J, Philippar U, et al: Simultaneous imaging of GFP, CFP and collagen in tumors in vivo using multiphoton microscopy. BMC Biotechnol 5: 14, 2005.

14. Wyckoff J, Wang W, Lin EY, et al: A paracrine loop between tumor cells and macrophages is required for tumor cell migration in mammary tumors. Cancer Res 64: 7022-7029, 2004.

15. Wyckoff JB, Wang Y, Lin EY, et al: Direct visualization of macrophage-assisted tumor cell intravasation in mammary tumors. Cancer Res 67: 2649-2656, 2007.

16. Xue C, Wyckoff J, Liang F, et al: Epidermal growth factor receptor overexpression results in increased tumor cell motility in vivo coordinately with enhanced intravasation and metastasis. Cancer Res 66: 192-197, 2006.

17. Nakagawa J, Saio M, Tamakawa N, et al: TNF expressed by tumor-associated macrophages, but not microglia, can eliminate glioma. Int J Oncol 30: 803-811, 2007.

18. Stout RD, Jiang C, Matta B, et al: Macrophages sequentially change their functional phenotype in response to changes in microenvironmental influences. J Immunol 175: 342-349, 2005.

19. Stout RD and Suttles J: Functional plasticity of macrophages: reversible adaptation to changing microenvironments. J Leukoc Biol 76: 509-513, 2004.

20. Dominguez PM and Ardavin C: Differentiation and function of mouse monocyte-derived dendritic cells in steady state and inflammation. Immunol Rev 234: 90-104, 2010.

21. Nonaka K, Saio M, Suwa T, et al: Skewing the Th cell phenotype toward Th1 alters the maturation of tumor-infiltrating mononuclear phagocytes. J Leukoc Biol 84: 679-688, 2008.

22. Umemura N, Saio M, Suwa T, et al: Tumor-infiltrating myeloidderived suppressor cells are pleiotropic-inflamed monocytes/ macrophages that bear M1- and M2-type characteristics. J Leukoc Biol 83: 1136-1144, 2008.

23. Mantovani A, Sozzani S, Locati M, et al: Macrophage polarization: tumor-associated macrophages as a paradigm for polarized M2 mononuclear phagocytes. Trends Immunol 23: 549-555, 2002.

24. Banyer JL and Hapel AJ: Myb-transformed hematopoietic cells as a model for monocyte differentiation into dendritic cells and macrophages. J Leukoc Biol 66: 217-223, 1999.

25. Gordon S: Alternative activation of macrophages. Nat Rev Immunol 3: 23-35, 2003.

26. Biswas SK, Gangi L, Paul S, et al: A distinct and unique transcriptional program expressed by tumor-associated macrophages (defective NF-kappaB and enhanced IRF-3/STAT1 activation). Blood 107: 2112-2122, 2006.

27. Hagemann T, Lawrence T, McNeish I, et al: 'Re-educating' tumor-associated macrophages by targeting NF-kappaB. J Exp Med 205: 1261-1268, 2008.

28. Conti L and Gessani S: GM-CSF in the generation of dendritic cells from human blood monocyte precursors: recent advances. Immunobiology 213: 859-870, 2008.

29. Ferlazzo G, Klein J, Paliard X, et al: Dendritic cells generated from $\mathrm{CD} 34^{+}$progenitor cells with flt3 ligand, c-kit ligand, GM-CSF, IL-4, and TNF-alpha are functional antigen-presenting cells resembling mature monocyte-derived dendritic cells. J Immunother 23: 48-58, 2000.

30. Chapuis F, Rosenzwajg M, Yagello M, et al: Differentiation of human dendritic cells from monocytes in vitro. Eur J Immunol 27: 431-441, 1997. 
31. Fujii S, Takayama T, Asakura M, et al: Dendritic cell-based cancer immunotherapies. Arch Immunol Ther Exp (Warsz) 57: 189-198, 2009.

32. Gilboa E: DC-based cancer vaccines. J Clin Invest 117: 1195-1203, 2007.

33. Hamilton JA: CSF-1 and cell cycle control in macrophages. Mol Reprod Dev 46: 19-23, 1997.

34. Naito M, Umeda S, Takahashi K, et al: Macrophage differentiation and granulomatous inflammation in osteopetrotic mice (op/op) defective in the production of CSF-1. Mol Reprod Dev 46: 85-91, 1997.

35. Jose MD, Le MY, Atkins RC, et al: Blockade of macrophage colony-stimulating factor reduces macrophage proliferation and accumulation in renal allograft rejection. Am J Transplant 3 : 294-300, 2003

36. Irvine KM, Burns CJ, Wilks AF, et al: A CSF-1 receptor kinase inhibitor targets effector functions and inhibits pro-inflammatory cytokine production from murine macrophage populations. FASEB J 20: 1921-1923, 2006.

37. Yu W, Chen J, Xiong Y, et al: CSF-1 receptor structure/function in MacCsf1r-/- macrophages: regulation of proliferation, differentiation, and morphology. J Leukoc Biol 84: 852-863, 2008.

38. Le MY, Tesch GH, Hill PA, et al: Macrophage accumulation at a site of renal inflammation is dependent on the M-CSF/c-fms pathway. J Leukoc Biol 72: 530-537, 2002.

39. Valk E, Zahn S, Knop J, et al: JAK/STAT pathways are not involved in the direct activation of antigen-presenting cells by contact sensitizers. Arch Dermatol Res 294: 163-167, 2002.

40. Jackson SH, Yu CR, Mahdi RM, et al: Dendritic cell maturation requires STAT1 and is under feedback regulation by suppressors of cytokine signaling. J Immunol 172: 2307-2315, 2004.

41. Welte T, Koch F, Schuler G, et al: Granulocyte-macrophage colony-stimulating factor induces a unique set of STAT factors in murine dendritic cells. Eur J Immunol 27: 2737-2740, 1997.
42. Chomarat P, Banchereau J, Davoust J, et al: IL-6 switches the differentiation of monocytes from dendritic cells to macrophages. Nat Immunol 1: 510-514, 2000.

43. Kusmartsev S and Gabrilovich DI: STAT1 signaling regulates tumor-associated macrophage-mediated T cell deletion. J Immunol 174: 4880-4891, 2005.

44. Biswas SK and Lewis CE: NF-kappaB as a central regulator of macrophage function in tumors. J Leukoc Biol 88: 877-884, 2010.

45. Hagemann T, Biswas SK, Lawrence T, et al: Regulation of macrophage function in tumors: the multifaceted role of NF-kappaB Blood 113: 3139-3146, 2009.

46. Saccani A, Schioppa T, Porta C, et al: p50 nuclear factor-kappaB overexpression in tumor-associated macrophages inhibits M1 inflammatory responses and antitumor resistance. Cancer Res 66: 11432-11440, 2006.

47. Biswas SK, Sica A and Lewis CE: Plasticity of macrophage function during tumor progression: regulation by distinct molecular mechanisms. J Immunol 180: 2011-2017, 2008.

48. Nicoletti A, Caligiuri G, Tornberg I, et al: The macrophage scavenger receptor type A directs modified proteins to antigen presentation. Eur J Immunol 29: 512-521, 1999.

49. Ceddia MA and Woods JA: Exercise suppresses macrophage antigen presentation. J Appl Physiol 87: 2253-2258, 1999.

50. Kondo H, Hazama S, Kawaoka T, et al: Adoptive immunotherapy for pancreatic cancer using MUC1 peptide-pulsed dendritic cells and activated T lymphocytes. Anticancer Res 28: 379-387, 2008.

51. Bucala R and Donnelly SC: Macrophage migration inhibitory factor: a probable link between inflammation and cancer. Immunity 26: 281-285, 2007

52. Mantovani A, Allavena P and Sica A: Tumour-associated macrophages as a prototypic type II polarised phagocyte population: role in tumour progression. Eur J Cancer 40: 1660-1667, 2004. 